\title{
EnRICH: Extraction and Ranking using Integration and Criteria Heuristics
}

Xia Zhang ${ }^{1,3}$, M Heather West Greenlee ${ }^{2,3,4^{*}}$ and Jeanne M Serb ${ }^{1,3}$

\begin{abstract}
Background: High throughput screening technologies enable biologists to generate candidate genes at a rate that, due to time and cost constraints, cannot be studied by experimental approaches in the laboratory. Thus, it has become increasingly important to prioritize candidate genes for experiments. To accomplish this, researchers need to apply selection requirements based on their knowledge, which necessitates qualitative integration of heterogeneous data sources and filtration using multiple criteria. A similar approach can also be applied to putative candidate gene relationships. While automation can assist in this routine and imperative procedure, flexibility of data sources and criteria must not be sacrificed. A tool that can optimize the trade-off between automation and flexibility to simultaneously filter and qualitatively integrate data is needed to prioritize candidate genes and generate composite networks from heterogeneous data sources.

Results: We developed the java application, EnRICH (Extraction and Ranking using Integration and Criteria Heuristics), in order to alleviate this need. Here we present a case stūdy in which we used EnRICH to integrate and filter multiple candidate gene lists in order to identify potential retinal disease genes. As a result of this procedure, a candidate pool of several hundred genes was narrowed down to five candidate genes, of which four are confirmed retinal disease genes and one is associated with a retinal disease state.

Conclusions: We developed a platform-independent tool that is able to qualitatively integrate multiple heterogeneous datasets and use different selection criteria to filter each of them, provided the datasets are tables that have distinct identifiers (required) and attributes (optional). With the flexibility to specify data sources and filtering criteria, EnRICH automatically prioritizes candidate genes or gene relationships for biologists based on their specific requirements. Here, we also demonstrate that this tool can be effectively and easily used to apply highly specific user-defined criteria and can efficiently identify high quality candidate genes from relatively sparse datasets.
\end{abstract}

Keywords: Qualitative integration, High-throughput data, Heterogeneous data, Network, Network visualization, Candidate prioritization

\section{Background}

Hundreds to thousands of candidate genes, or genes of interest, can now be generated from a single experiment utilizing high throughput screening technologies. However, the number of candidate genes that can be experimentally studied in-depth is often constrained by time and cost. Therefore, prioritization of candidate genes is a critical step in the experimental process. Approaches to identify 'the most promising' candidates are becoming increasingly

\footnotetext{
* Correspondence: mheather@iastate.edu

${ }^{2}$ Department of Biomedical Sciences, 2008 Veterinary Medicine, lowa State University, Ames, IA 50010, USA

${ }^{3}$ Interdepartmental Genetics Program, lowa State University, Ames, lowa, USA Full list of author information is available at the end of the article
}

more sophisticated. For example, when microarray studies were initially reported, 'the most promising' candidates were often the most differentially expressed and could be obtained by a simple ranking of candidates based on fold change. As more data has become available, biologists have begun to look for ways [1-4] to use multiple data sources to increase the accuracy of candidate gene prioritization. Some tools have already been developed to address this need [5-11]. These tools prioritize candidates by their similarity to genes already known to be important for a particular biological process (e.g., genes known to regulate cell cycle in yeast). Multiple data sources including published literature, gene sequence, functional annotation, etc. can be considered when comparing the similarity of candidates to

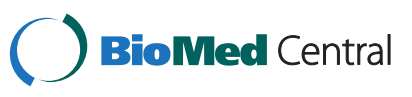


'known genes'. These tools [5-11] have made important progress towards the problem of candidate prioritization. However, these tools use data queried from predetermined sources, such as public databases, and include embedded criteria. Thus, these software packages have limited utility.

Biologists, with expertise in a given area, generally already have a list of criteria that could be applied to identify high quality candidates. Likely, for a given set of experiments and resulting datasets, the best candidates may satisfy one set of criteria in one dataset and a separate set of criteria in another dataset. Currently, there is no tool that allows simultaneous consideration of heterogeneous datasets to identify candidates that satisfy multiple criteria. This problem does not only relate to candidate genes, but also to putative relationships between genes in networks.

Putative gene relationships can be inferred from many heterogeneous sources (e.g., physical interactions, genetic interactions, expression correlation and interactions predicted by computational models). While each of these relationships from a given dataset should be interpreted differently (and subject to very different criteria), the ability to easily hypothesize gene relationships based on their meeting appropriate criteria in multiple datasets is an attractive prospect. This task not only calls for an automated filtering and integration tool, but also demands great flexibility of data sources and the ability to set filtering criteria. Finally, for proper interpretation, visualization of the resulting network must facilitate inspection by 1 ) retaining the original data sources of each putative relationship and 2) providing a mechanism to easily manage the size of the displayed network. While some tools have been developed to generate composite networks from multiple data sources (e.g., the Cytoscape [12] plugin CABIN [13], GraphWeb [14] and GeneMania [15]), they do not fully address the problems stated above. For example, CABIN supports only one filter for a single source network and thus multiple criteria cannot be applied. GraphWeb [14] does not support filtering by user-defined criteria and interactive network visualization. GeneMania [15] helps to predict the function of a set of input genes by utilizing functional association data to generate a functional relevant network, but does not address integration of userdetermined data and filtration with user-defined criteria.

We identified the need for a tool that is able to: 1) filter individual datasets using appropriate criteria and then integrate them to prioritize candidates that meet the criteria in multiple datasets; 2) allow users to define the most appropriate datasets and filtering criteria; and 3) provide an interactive visualization to facilitate the generation of an integrated network with a manageable size and connectedness. To address the open demand of filtering and qualitative integration of heterogeneous datasets, we have developed a stand-alone, portable and flexible java application with its own user-interactive visualization. EnRICH (Extraction and Ranking using Integration and

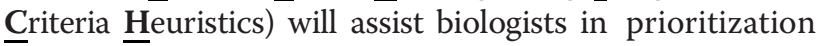
of genes and gene relationships from heterogeneoussource data.

\section{Implementation}

EnRICH was implemented in Java (SE 6 JDK). EnRICH visualization was written in Processing (http://processing. org/), an open-source programming language to create images, animation and interactions. The separation of non-visual and visual modules of EnRICH lays a flexible foundation for future development and provides the user easy access to both the text and visual output results.

\section{Design}

The objectives of EnRICH are firstly to provide a tool for integration of multiple or heterogeneous data sets to prioritize candidate molecules that fulfill user-defined criteria, and secondly to make the integration process flexible and simple for biologists who have little programming skill. Our aim-oriented design principles are 1) user-defined data sources and criteria, 2) simplicity which allows straightforward application of user-defined criteria to filter userdefined datasets, and 3) platform independence.

The overall architecture of EnRICH is reflected in its workflow-like graphical user interface (GUI) (Figure 1). The first component (numbered as step 1 in the GUI) accepts a single file or a directory of files as input data and lists all files that can be selected for analysis. The second component (numbered as step 2 in the GUI) allows the user to display the selected file as a table and edit the table. The third component (numbered as step 3 in the GUI) enables the user to specify filtering criteria for each attribute of the selected file. The fourth component (numbered as step 4 in the GUI) displays all uploaded files for the user to customize an integration pool. It also provides the user running options on whether to apply filters that are already specified in step 3 . The fifth component is a dialog window, which appears when the integration run is finished, and gives the user the option to save or visualize the result. For network data, EnRICH has an additional visualization component where the user can do an interactive visual analysis of the integrated network.

\section{Input data}

The current version of EnRICH accepts two types of data: list and network. A list is a set of elements that could be genes, proteins, etc., which have their own unique identification code or name. List data can come from a large variety of sources. For example, a list of genes can be differentially expressed genes (DEGs) from the analysis of a microarray experiment, genes identified by genome-wide association mapping, or genes retrieved from a database 


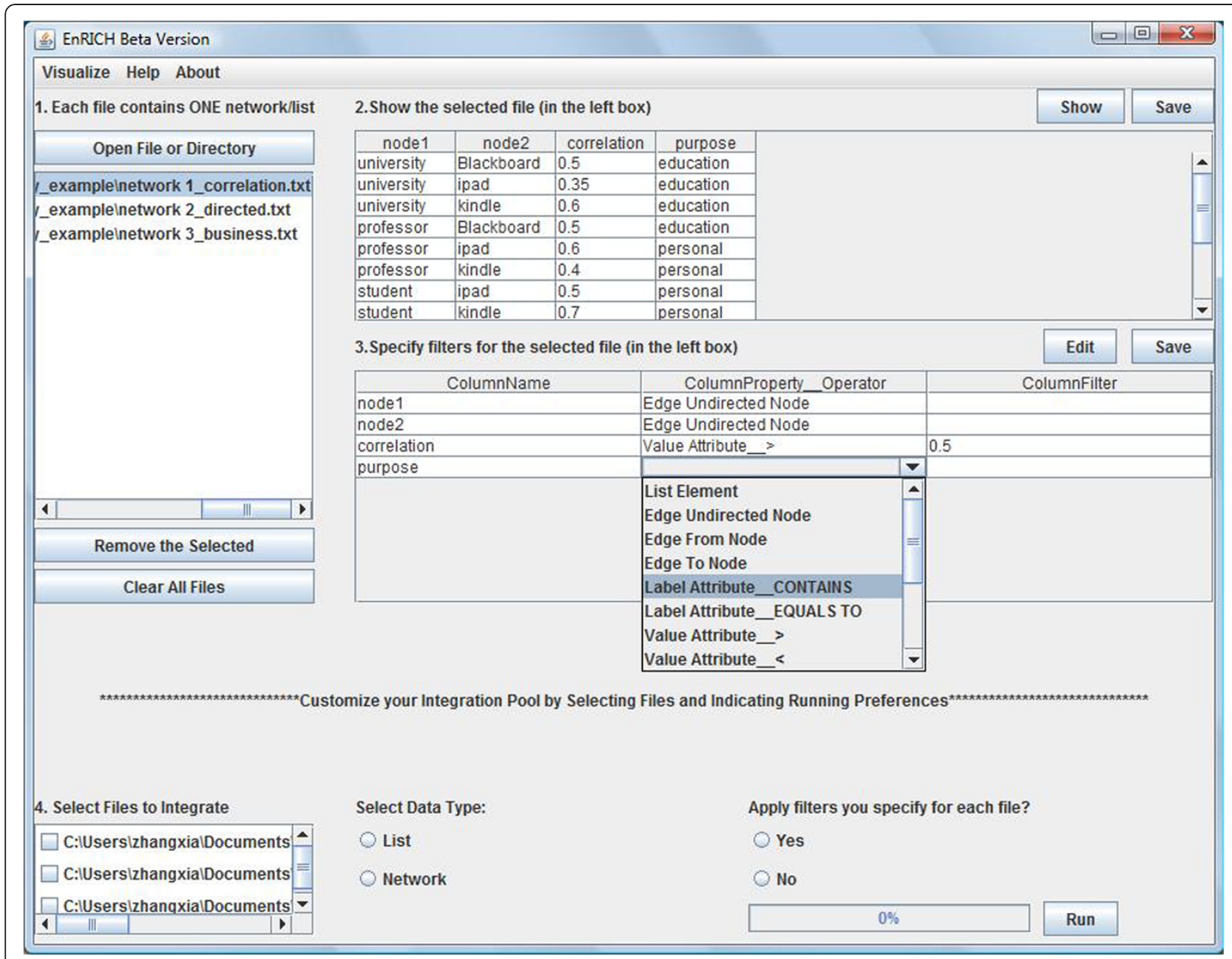

Figure 1 EnRICH graphical user interface. There are four major components in the user interface, which are numbered as 1, 2, 3 and 4. Component 1: upload input data; Component 2: browse or edit the selected file; Component 3: specify filtering criteria of the selected file; Component 4: select files to define integration pool.

query. Each list member may have one or more attributes. For example, each gene in a list of DEGs has its own significance value, functional annotation, etc. For EnRICH, list data is represented as a named matrix that is composed of one column of elements and zero to multiple attribute columns. Attributes can either be value attributes that will be taken as mathematical values or label attributes treated as tags.

A network is a set of nodes that are interconnected by edges representing particular relationships between nodes. Like list data, network data can originate from heterogeneous sources including yeast two-hybrid experiments, computational or statistical inferences, literature summaries or database queries. Although there are several standard languages or formats for network representation, we assume that biologists may not be familiar with those standards. Thus, EnRICH applies a popular node-pair/edge list format as the input format for network data, where an edge is denoted by the pair of nodes it connects. In the matrix format, network edges are represented by two columns of node names. Like list data, network edges may have values and label attributes. Accordingly, a network is a named matrix consisting of two columns of nodes and zero to multiple attribute columns. EnRICH allows blank fields in the attribute column when data are missing.

\section{Running mode}

EnRICH runs in two modes: undefined (without filters) and defined (using specific criteria to filter attributes). The undefined mode simply ignores the attributes of networks or lists. Each list or network is considered as a source, and all sources will be merged together. The defined mode simultaneously considers integration of networks or lists as well as user-defined criteria (which filters out elements that do not meet the criteria) over each network or list. For both types of running modes, candidates (edges of a 
network or elements of a list) are ranked by their reoccurrence across all sources after integration. The filtering process is completely user-defined. Because the filter is totally attribute-based, the user sets filters most appropriate for their biological question, which may include a combination of filters for each attribute, and even multiple filters for multiple attributes. For example, two of the comparison operators $(<,<=,>,>=,==)$ applied at the same time can be used to set a cutoff range for value attributes or several tags can be used (with an OR operator between them) when the user wants to select multiple label values (e.g. two annotations) for one attribute. When there are multiple attributes, multiple filters (with an AND operator between them) can be applied simultaneously.

\section{Text output}

EnRICH saves output results as a tab-delimited text file. In the output text file, the user can see what files were integrated, which filters were applied to each file, and the result. For list data, the result is a table, which consists of three columns: the label of an element, its reoccurrence across all lists, and names of source-lists. For network data, the result includes four tables: node statistics, edge statistics, nodes, and edges. The node degree reveals topological importance of the node, so the table of node statistics contains two columns, one column is the node degree (the number of connections a single node has) and the other is the number of nodes that are greater than or equal to $(>=)$ this node degree. For the table of edge statistics, one column is edge reoccurrence (the number of times a single edge is recovered across all datasets) and the other is the number of edges that have an edge reoccurrence that are greater than or equal to $(>=)$ this edge reoccurrence. The table of nodes and the table of edges are quite similar. Each has a column of nodes/edges, their reoccurrence, and source-networks. The only difference between the node and edge tables is that a node is represented by the node label and an edge is denoted as two node labels. The table of edges is a tab-delimited data table composed of several columns such as node label name, edge reoccurrence and source. Therefore, if desired, the user can directly copy or import them into another network visualization tool such as Cytoscape [12,16].

\section{Visualization}

EnRICH enables an interactive visual analysis of the integrated network without depending on a third-party visualization software. EnRICH network visualization consists of two components for user interaction: the integrated network and the plot of network statistics (Figure 2). In the integrated network, an undirected edge is drawn as a blue line while a directed edge is drawn as a pink line with a pink arrow to indicate the direction of the interaction (e.g. transcriptional regulation). A blue line with a pink arrow is used to denote merged undirected and directed edges. All edges and nodes can be repositioned, without changing connections, by clicking and then dragging the item on the screen. In addition, the user can click to show or dissipate node labels and edge sources at the node- and edge-specific level, instead of the whole network level. The plot component has two plots: 1) the number of nodes vs. node degree plot and 2) the number of edges vs. edge-reoccurrence plot. The number of nodes and the number of edges are two aspects of the network size, while the node degree reveals topological importance of node. Edge reoccurrence is the number of times the edge is recovered in different data sources, which implies the reliability of an edge. In conjunction, the two plots are used to balance the visualization of network size and quality. All data points in the two plots are clickable to re-draw the integrated network at the selected level of node degree or edge reoccurrence. This interactive plot gives the user an easily visible comparison of node degree, edge reoccurrence and network size, and allows the user to simultaneously visualize the network at corresponding levels. EnRICH also allows the exportation of the network as an image file in TIFF format, which is widely supported.

\section{Result and discussion Application of EnRICH}

Retinal disease genes are genes that, when knocked out or mutated, cause retinal degeneration (https://sph.uth.tmc. $\mathrm{edu} / \mathrm{retnet} / \mathrm{disease} . \mathrm{htm})$. The identification of retinal disease genes is a major goal of retinal degenerative disease research and as part of this effort, there have been a significant number of experiments that describe transcriptional changes during normal retinal development [17-23]. Here, we present a case study in which we use EnRICH to integrate multiple gene lists to identify potential retinal disease genes.

$\mathrm{Nrl}$ [24-27] is a retinal disease gene that is associated with the retinal degenerative disease enhanced s-cone syndrome [28]. When $\mathrm{Nrl}$ is mutated, the resulting phenotype is an abundance of s-cone photoreceptors at the expense of rod photoreceptor differentiation [25,29], leading to the eventual death of all photoreceptors. During normal development, $\mathrm{Nrl}$ influences the cone versus the rod cell fate decision by activating rod-specific genes, including the genes Rho and Nr2e3 [30]. Rho [31-33] is a rod-specific gene, the mutation of which leads to rod photoreceptor cell death and retinal degeneration. Nr2e3 $[34,35]$ is also essential during retinal development, as it promotes the expression of rod-specific genes (including Rho) and represses the expression of cone-specific genes in rods. The mutation of $\mathrm{Nr} 2 \mathrm{e} 3$ also causes enhanced s-cone syndrome [36]. Based on the known 


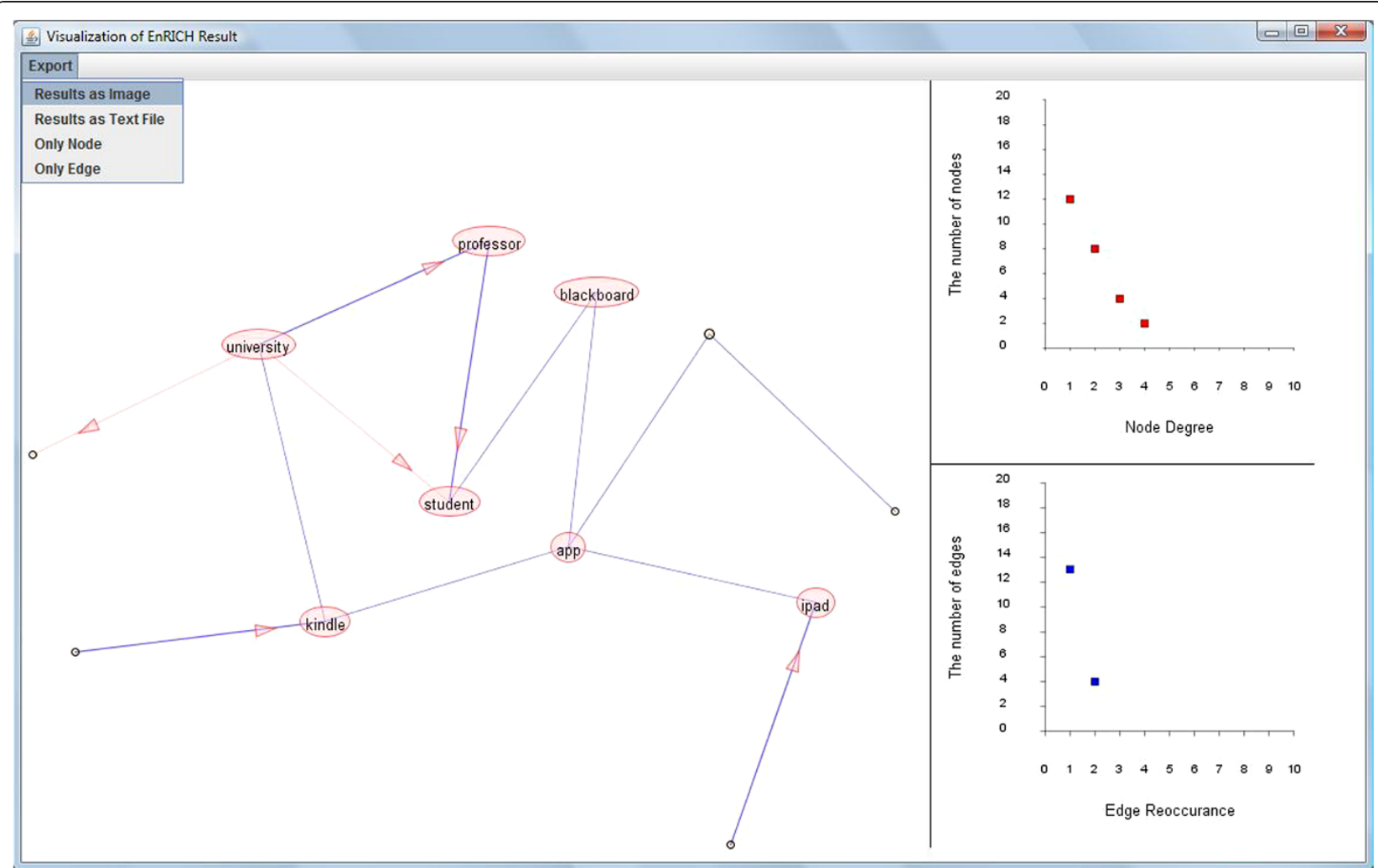

Figure 2 EnRICH visualization window. Left: integrated network from synthesizing data. Circles represent nodes, while circle size represents node degree (the number of connections one node has). Lines represent edges of a network and line stroke represents the amount of edge reoccurrence (the number of times one edge is recovered across data sources). Undirected edges are represented by blue lines, while directed edges are pink lines with pink arrows. The merged edge of undirected and directed data is denoted by blue line with a pink arrow. Right: top panel is the statistical plot of node degree vs. the number of nodes. Bottom panel is the statistical plot of edge reoccurrence vs. the number of edges. In the software, all data points in the two plots are clickable to update visualization of the integrated network.

regulatory relationships between these three disease genes and their importance for normal photoreceptor development, we rationalize that the behavior of these genes would make good criteria to identify additional retinal disease genes.
Using these assumptions, we defined the following criteria to identify retinal disease genes: 1 ) candidates must be highly co-expressed with $\mathrm{Nrl}, \mathrm{Nr} 2 \mathrm{e} 3$ and $\mathrm{Rho}$ during rod photoreceptor development of wild-type mice; and 2) candidates must be disregulated when $\mathrm{Nrl}$ is knocked

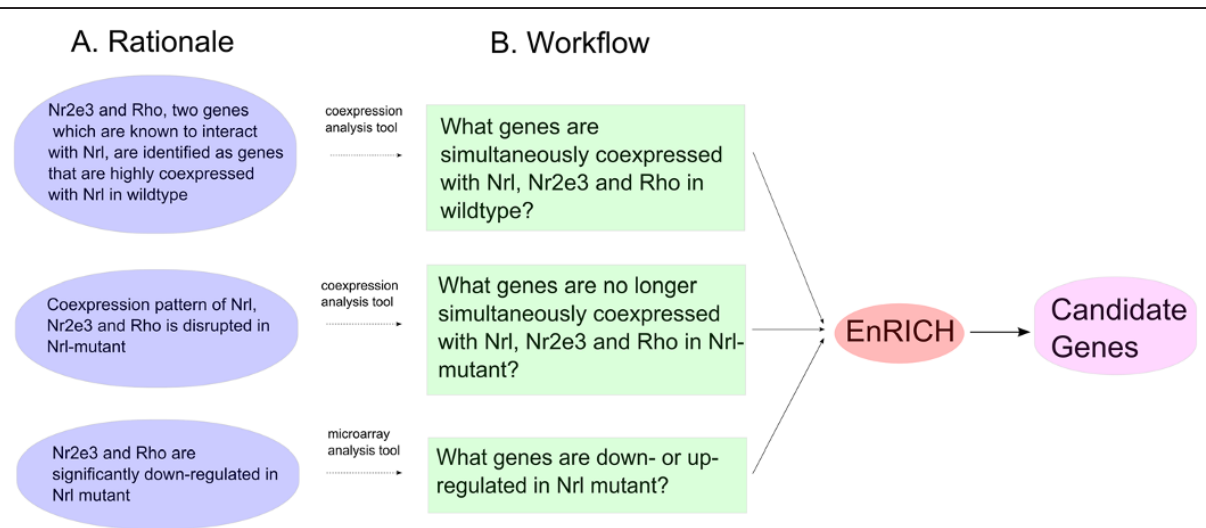

Figure 3 Case study workflow. A. Rationale illustrates our current knowledge of Nrl, Nr2e3 and Rho and their behaviors in the dataset we analyzed. B. Workflow displays the steps that we must go through in order to apply our criteria to identify candidate genes. These steps are executed by EnRICH to obtain candidate genes. Workflow is the implementation of the investigation process based on the rationale (A). 
out (as Nr2e3 and Rho are). With these criteria in mind, we decided to use a microarray dataset [17] (GSE4051), which profiles gene expression in isolated rod photoreceptors at multiple developmental stages (E16, P2, P6, xP10, 4-weeks) in both $\mathrm{Nrl}$-knockout and wild-type mice. In these microarrays, we confirmed that $N r 2 e 3$ and Rho are highly co-expressed with $\mathrm{Nrl}$ in wildtype and are no longer co-expressed in the $\mathrm{Nrl}$ mutant.

According to the corresponding workflow (Figure 3), we prepared, and subsequently integrated, three types of gene lists which are: Type 1) Genes that are co-expressed with $\mathrm{Nrl}, \mathrm{Nr} 2 \mathrm{e} 3$ and Rho in developing wild type rod photoreceptors; Type 2) Genes that are co-expressed with $\mathrm{Nrl}$, Nr2e3 and Rho in developing photoreceptors isolated from Nrl-mutant retinas; and Type 3) Differentially expressed genes (DEGs) at each age when comparing gene expression in wild-type rod photoreceptors to $\mathrm{Nrl}$-knockout rods. Each list contained attributes that were used to apply criteria filters (i.e. pairwise correlations for type 1 and 2, age at which expression was up or down regulated for type 3). To carry out the workflow, we first specified filtering criteria for each list. This is a key element of EnRICH, where users can simultaneously query multiple datasets to generate an 'integrated result'. For this experiment, eight list datasets were integrated. The filtering criterion for six of the lists was an absolute value of the correlation coefficient greater than 0.9 , while the filtering criterion for the two differentially expressed gene lists was the developmental time points P6 and P10 (for criteria on each single list, see Additional file 1: Table S1). Candidates that satisfied these filtering criteria in eight lists were identified as the highest priority candidates. All the lists were prepared from by standard analyses of the dataset GSE 4051 (calculation of co-expression coefficients within a genotype and differentially expressed genes between genotypes).

The execution of our workflow generated five candidate genes (see Additional file 2: Table S2) from an initial pool of 272 unique differentially expressed genes (see Additional file 3). Based on a literature/database search, four of our five candidate genes (pde6b, gnb1, gucala and cgnal) are confirmed retinal disease genes [37-46], and the fifth gene (kcne2) has been shown to be up regulated during a neuroinflamatory response in the retinas of diabetic rats [47], making it a reasonable candidate for a disease gene as well. Thus, in our example analysis to identify disease genes, $80 \%$ of our candidates are known disease genes, while the remaining candidate has a demonstrated tie to the diseased retina, and is therefore a high quality candidate. Using a Fisher test we also concluded that retinal disease genes are significantly overrepresented in the genes prioritized by EnRICH, compared with genes not prioritized by EnRICH (see Additional file 3).
Our case study demonstrates that a well-conceived data integration and criteria-based filtration, as implemented in EnRICH, can effectively identify a limited number of high quality candidate genes for careful hypothesis-based investigation. Conversely, if the number of candidates returned is too small, slight adjustments in the filtration criteria may be easily made to generate a larger, while still reasonablysized, candidate pool.

\section{Conclusions}

EnRICH is a free java application which can qualitatively integrate results from large, heterogeneous data sources while simultaneously applying filters to each of them. It allows the user to define data sources, and to integrate them as well as specify multiple sorting criteria specific to each data source. It provides interactive network visualization tool for the user to identify an integrated network with a desirable balance between network size and quality. With EnRICH, biologists have an automated yet flexible integration tool to carry out their data analysis and effectively prioritize candidate genes for further investigation.

\section{Availability and requirements}

Project name: EnRICH (see Additional file 4: for the jar file of EnRICH program).

Project home page: http://xiazhang.public.iastate.edu/ or the software category on the lab homepage http:// serb.public.iastate.edu.

Operating system(s): platform-independent.

Programming language: Java.

Other requirements: Java 1.4.2 or higher.

License: GNU General Public License.

Any restrictions to use by non-academics: NO.

\section{Additional files}

Additional file 1: Gene lists and their filtration criteria prior to integration. This file includes a supplementary table that displays names and descriptions of gene lists for integration in case study and some further explanation on the sources of gene lists.

Additional file 2: Gene candidates resulting from the EnRICH filtration and prioritization analyses. This file is the supplementary table of the five gene candidates from the prioritization by using $\mathrm{EnRICH}$ in the case study.

Additional file 3: Description of data processing. This file includes detail description of data pre-processing for case study, and analysis of the significance of case study result.

Additional file 4: EnRICH program. This file is the archive file of EnRICH which aggregates Java class files and associated metadata and resources.

\section{Competing interest}

The author(s) declare that they have no competing interests.

\section{Authors' contributions}

$X Z$, JMS and MHWG conceived and designed this software, and drafted this manuscript. XZ coded this software and conducted case study presented in this manuscript. All authors read and approved the final manuscript. 


\section{Acknowledgements}

The authors wish to thank Guan Wang and Fadi Towfic for their helpful comments on this software. Financial support was provided by the Center for Integrative Animal Genomics at lowa State University to JMS and MHWG.

\section{Author details}

${ }^{1}$ Department of Ecology, Evolution, and Organismal Biology, lowa State University, Ames, lowa, USA. ${ }^{2}$ Department of Biomedical Sciences, 2008 Veterinary Medicine, lowa State University, Ames, IA 50010, USA ${ }^{3}$ Interdepartmental Genetics Program, lowa State University, Ames, lowa, USA. ${ }^{4}$ Bioinformatics and Computational Biology Program, lowa State University, Ames, lowa, USA.

Received: 11 April 2012 Accepted: 7 January 2013

Published: 15 January 2013

\section{References}

1. Zheng S, Tansey WP, Hiebert SW, Zhao Z: Integrative network analysis identifies key genes and pathways in the progression of hepatitis $C$ virus induced hepatocellular carcinoma. BMC Med Genomics 2011, 4:62.

2. Aragues R, Sander C, Oliva B: Predicting cancer involvement of genes from heterogeneous data. BMC Bioinforma 2008, 9:172.

3. Kao CF, Fang YS, Zhao Z, Kuo PH: Prioritization and evaluation of depression candidate genes by combining multidimensional data resources. PLoS One 2011, 6(4):e18696.

4. Bare JC, Koide T, Reiss DJ, Tenenbaum D, Baliga NS: Integration and visualization of systems biology data in context of the genome. BMC Bioinforma 2010, 11:382

5. Tranchevent LC, Capdevila FB, Nitsch D, De Moor B, De Causmaecker P, Moreau $Y$ : A guide to web tools to prioritize candidate genes. Brief Bioinform 2010, 12(1):22-32.

6. Chen J, Bardes EE, Aronow BJ, Jegga AG: ToppGene Suite for gene list enrichment analysis and candidate gene prioritization. Nucleic Acids Res 2009, 37(Web Server issue):W305-311.

7. Adie EA, Adams RR, Evans KL, Porteous DJ, Pickard BS: SUSPECTS: enabling fast and effective prioritization of positional candidates. Bioinformatics 2006, 22(6):773-774

8. Fontaine JF, Priller F, Barbosa-Silva A, Andrade-Navarro MA: Genie: literature-based gene prioritization at multi genomic scale. Nucleic Acids Res 2011, 39(Web Server issue):W455-461.

9. Nitsch D, Tranchevent LC, Goncalves JP, Vogt JK, Madeira SC, Moreau Y: PINTA: a web server for network-based gene prioritization from expression data. Nucleic Acids Res 2011, 39(Web Server issue):W334-338.

10. Tranchevent LC, Barriot R, Yu S, Van Vooren S, Van Loo P, Coessens B, De Moor B, Aerts S, Moreau Y: ENDEAVOUR update: a web resource for gene prioritization in multiple species. Nucleic Acids Res 2008, 36(Web Server issue):W377-384

11. Doncheva NT, Kacprowski T, Albrecht M: Recent approaches to the prioritization of candidate disease genes. Wiley Interdiscip Rev Syst Biol Med 2012, 4(5):429-442

12. Killcoyne S, Carter GW, Smith J, Boyle J: Cytoscape: a community-based framework for network modeling. Methods Mol Biol 2009, 563:219-239.

13. Singhal $M$, Domico K: CABIN: collective analysis of biological interaction networks. Comput Biol Chem 2007, 31(3):222-225.

14. Reimand J, Tooming L, Peterson H, Adler P, Vilo J: GraphWeb: mining heterogeneous biological networks for gene modules with functional significance. Nucleic Acids Res 2008, 36(Web Server issue):W452-459.

15. Mostafavi S, Ray D, Warde-Farley D, Grouios C, Morris Q: GeneMANIA: a real-time multiple association network integration algorithm for predicting gene function. Genome Biol 2008, 9(Suppl 1):S4.

16. Kohl M, Wiese S, Warscheid B: Cytoscape: software for visualization and analysis of biological networks. Methods Mol Biol 2011, 696:291-303.

17. Akimoto M, Cheng H, Zhu D, Brzezinski JA, Khanna R, Filippova E, Oh EC, Jing $Y$, Linares $J L$, Brooks $M$, et al: Targeting of GFP to newborn rods by $\mathrm{Nrl}$ promoter and temporal expression profiling of flow-sorted photoreceptors. Proc Natl Acad Sci USA 2006, 103(10):3890-3895.

18. Dorrell MI, Aguilar E, Weber C, Friedlander M: Global gene expression analysis of the developing postnatal mouse retina. Invest Ophthalmol Vis Sci 2004, 45(3):1009-1019.
19. Liu J, Wang J, Huang Q, Higdon J, Magdaleno S, Curran T, Zuo J: Gene expression profiles of mouse retinas during the second and third postnatal weeks. Brain Res 2006, 1098(1):113-125.

20. Trimarchi JM, Stadler MB, Roska B, Billings N, Sun B, Bartch B, Cepko CL: Molecular heterogeneity of developing retinal ganglion and amacrine cells revealed through single cell gene expression profiling. J Comp Neurol 2007, 502(6):1047-1065.

21. Feng Y, Wang Y, Li L, Wu L, Hoffmann S, Gretz N, Hammes HP: Gene expression profiling of vasoregression in the retina--involvement of microglial cells. PLoS One 2011, 6(2):e16865.

22. Leung YF, Dowling JE: Gene expression profiling of zebrafish embryonic retina. Zebrafish 2005, 2(4):269-283.

23. Kamphuis W, Dijk F, van Soest S, Bergen AA: Global gene expression profiling of ischemic preconditioning in the rat retina. Mol Vis 2007 13:1020-1030

24. Rehemtulla A, Warwar R, Kumar R, Ji X, Zack DJ, Swaroop A: The basic motif-leucine zipper transcription factor $\mathrm{Nrl}$ can positively regulate rhodopsin gene expression. Proc Natl Acad Sci USA 1996, 93(1):191-195.

25. Mears AJ, Kondo M, Swain PK, Takada Y, Bush RA, Saunders TL, Sieving PA, Swaroop A: Nrl is required for rod photoreceptor development. Nat Genet 2001, 29(4):447-452.

26. Cheng H, Aleman TS, Cideciyan AV, Khanna R, Jacobson SG, Swaroop A: In vivo function of the orphan nuclear receptor NR2E3 in establishing photoreceptor identity during mammalian retinal development. Hum Mol Genet 2006, 15(17):2588-2602.

27. Oh EC, Cheng H, Hao H, Jia L, Khan NW, Swaroop A: Rod differentiation factor NRL activates the expression of nuclear receptor NR2E3 to suppress the development of cone photoreceptors. Brain Res 2008, 1236:16-29.

28. Hood DC, Cideciyan AV, Roman AJ, Jacobson SG: Enhanced S cone syndrome: evidence for an abnormally large number of $\mathrm{S}$ cones. Vision Res 1995, 35(10):1473-1481.

29. Daniele LL, Lillo C, Lyubarsky AL, Nikonov SS, Philp N, Mears AJ, Swaroop A Williams DS, Pugh EN Jr: Cone-like morphological, molecular, and electrophysiological features of the photoreceptors of the Nrl knockout mouse. Invest Ophthalmol Vis Sci 2005, 46(6):2156-2167.

30. Zhang $X$, Serb JM, Greenlee MH: Mouse retinal development: a dark horse model for systems biology research. Bioinform Biol Insights 2011, 5:99-113.

31. Cideciyan AV, Hood DC, Huang Y, Banin E, Li ZY, Stone EM, Milam AH, Jacobson SG: Disease sequence from mutant rhodopsin allele to rod and cone photoreceptor degeneration in man. Proc Natl Acad Sci USA 1998 95(12):7103-7108

32. lakhine R, Chorna-Ornan I, Zars T, Elia N, Cheng Y, Selinger Z, Minke B, Hyde DR: Novel dominant rhodopsin mutation triggers two mechanisms of retinal degeneration and photoreceptor desensitization. J Neurosci 2004 24(10):2516-2526.

33. Pennesi ME, Nishikawa S, Matthes MT, Yasumura D, LaVail MM: The relationship of photoreceptor degeneration to retinal vascular development and loss in mutant rhodopsin transgenic and RCS rats. Exp Eye Res 2008, 87(6):561-570.

34. Chen J, Rattner A, Nathans J: The rod photoreceptor-specific nuclear receptor Nr2e3 represses transcription of multiple cone-specific genes. J Neurosci 2005, 25(1):118-129.

35. Peng GH, Ahmad O, Ahmad F, Liu J, Chen S: The photoreceptor-specific nuclear receptor $\mathrm{Nr2e} 3$ interacts with $\mathrm{Crx}$ and exerts opposing effects on the transcription of rod versus cone genes. Hum Mol Genet 2005 , 14(6):747-764

36. Corbo JC, Cepko CL: A hybrid photoreceptor expressing both rod and cone genes in a mouse model of enhanced S-cone syndrome. PLoS Genet 2005, 1(2):e11.

37. Tosi J, Davis RJ, Wang NK, Naumann M, Lin CS, Tsang SH: shRNA knockdown of guanylate cyclase 2e or cyclic nucleotide gated channel alpha 1 increases photoreceptor survival in a cGMP phosphodiesterase mouse model of retinitis pigmentosa. J Cell Mol Med 2011, 15(8):1778-1787.

38. Hart AW, McKie L, Morgan JE, Gautier P, West K, Jackson IJ, Cross SH: Genotype-phenotype correlation of mouse pde6b mutations. Invest Ophthalmol Vis Sci 2005, 46(9):3443-3450.

39. Jacobson SG, Sumaroka A, Aleman TS, Cideciyan AV, Danciger M, Farber DB: Evidence for retinal remodelling in retinitis pigmentosa caused by PDE6B mutation. Br J Ophthalmol 2007, 91(5):699-701. 
40. Tsang SH, Woodruff ML, Jun L, Mahajan V, Yamashita CK, Pedersen R, Lin CS, Goff SP, Rosenberg T, Larsen M, et al: Transgenic mice carrying the H258N mutation in the gene encoding the beta-subunit of phosphodiesterase- 6 (PDE6B) provide a model for human congenital stationary night blindness. Hum Mutat 2007, 28(3):243-254.

41. Mylvaganam GH, McGee TL, Berson EL, Dryja TP: A screen for mutations in the transducin gene GNB1 in patients with autosomal dominant retinitis pigmentosa. Mol Vis 2006, 12:1496-1498.

42. Michaelides M, Wilkie SE, Jenkins S, Holder GE, Hunt DM, Moore AT, Webster AR: Mutation in the gene GUCA1A, encoding guanylate cyclaseactivating protein 1, causes cone, cone-rod, and macular dystrophy. Ophthalmology 2005, 112(8):1442-1447.

43. Buch PK, Mihelec M, Cottrill P, Wilkie SE, Pearson RA, Duran Y, West EL, Michaelides M, Ali RR, Hunt DM: Dominant cone-rod dystrophy: a mouse model generated by gene targeting of the GCAP1/Guca1a gene. PLoS One 2011, 6(3):e18089.

44. Kitiratschky VB, Behnen P, Kellner U, Heckenlively JR, Zrenner E, Jagle H, Kohl $\mathrm{S}$, Wissinger B, Koch KW: Mutations in the GUCA1A gene involved in hereditary cone dystrophies impair calcium-mediated regulation of guanylate cyclase. Hum Mutat 2009, 30(8):E782-796.

45. Jiang L, Katz BJ, Yang Z, Zhao Y, Faulkner N, Hu J, Baird J, Baehr W, Creel DJ, Zhang $\mathrm{K}$ : Autosomal dominant cone dystrophy caused by a novel mutation in the GCAP1 gene (GUCA1A). Mol Vis 2005, 11:143-151.

46. Dryja TP, Finn JT, Peng YW, McGee TL, Berson EL, Yau KW: Mutations in the gene encoding the alpha subunit of the rod CGMP-gated channel in autosomal recessive retinitis pigmentosa. Proc Natl Acad Sci USA 1995, 92(22):10177-10181.

47. Brucklacher RM, Patel KM, VanGuilder HD, Bixler GV, Barber AJ, Antonetti DA, Lin CM, LaNoue KF, Gardner TW, Bronson SK, et al: Whole genome assessment of the retinal response to diabetes reveals a progressive neurovascular inflammatory response. BMC Med Genomics 2008, 1:26.

doi:10.1186/1752-0509-7-4

Cite this article as: Zhang et al:: EnRICH: Extraction and Ranking using Integration and Criteria Heuristics. BMC Systems Biology 2013 7:4.

\section{Submit your next manuscript to BioMed Central and take full advantage of:}

- Convenient online submission

- Thorough peer review

- No space constraints or color figure charges

- Immediate publication on acceptance

- Inclusion in PubMed, CAS, Scopus and Google Scholar

- Research which is freely available for redistribution

Submit your manuscript at www.biomedcentral.com/submit
() Biomed Central 